\title{
Incidencia de la Educación financiera de socios en la gestión financiera de una Cooperativa de Ahorro y Crédito
}

Juan Portilla Ladron De Guevara

juan.portilla@unsaac.edu.pe

ORCID ID: https://orcid.org/0000-0001-9981-807X

Universidad Nacional de San Antonio Abad

Alan Alain Huaman Auccapuri alan.huaman@unsaac.edu.pe

ORCID ID: https://orcid.org/0000-0001-9386-9618 Universidad Nacional de San Antonio Abad

Daniel Alejandro Linares Santos daniel.linares@unsaac.edu.pe

ORCID ID: https://orcid.org/0000-0001-8479-5805 Universidad Nacional de San Antonio Abad

Franz Marcelino Herrera Huaracha info@iidest.onmicrosoft.com

ORCID ID: https://orcid.org/0000-0002-7710-7629

RESUMEN

Instituto de Investigación y Desarrollo Estratégico SAC

En la actualidad en el ámbito de las finanzas, como parte de la administración del dinero y el capital, vienen a conformar un factor elemental para generar liquidez financiera que permita a las organizaciones cumplir con sus obligaciones. El objetivo radica en explicar la incidencia de la educación financiera de los socios en la gestión financiera de la Cooperativa de Ahorro y Crédito «Santo Domingo de Guzmán». El método se fundamenta en el análisis de datos transversales, caracterizado por socios de 22 a 75 años, con un total de 51961 socios activos; siendo seleccionados 245 socios, a través de un muestreo probabilístico sistemático. Los resultados muestran una dispersión de los resultados, respecto a la educación financiera, evidenciando dificultades en el conocimiento financiero, en el caso de la gestión financiera en todas sus dimensiones se tiene una dispersión respecto al manejo financiero en el proceso administrativo siendo moderadamente eficaz. En conclusión, la participación de los socios es fundamental para lograr una sólida liquidez financiera, permitiéndole cumplir todas las obligaciones contraídas, siendo el factor más importante el conocimiento financiero de los socios, esto contribuye a generar mayor comprensión del ámbito financiero y atender de sus responsabilidades contraídas.

Palabras clave: Cooperativas de ahorro y crédito, educación financiera, gestión financiera. 


\title{
Incidence of the financial education of partners in the financial management of a Savings and Credit Cooperative
}

\begin{abstract}
At present in the field of finance, as part of the management of money and capital, they come to form an elementary factor to generate financial liquidity that allows organizations to meet their obligations. The objective is to explain the incidence of the financial education of the members in the financial management of the Cooperative de Saving y Credit «Santo Domingo de Guzmán». The method is based on cross-sectional data analysis, characterized by members from 22 to 75 years old, with a total of 51961 active members; being selected 245 partners, through a systematic probability sampling. The results show a dispersion of the results, regarding financial education, showing difficulties in financial knowledge, in the case of financial management in all its dimensions there is a dispersion regarding financial management in the administrative process being moderately effective. In conclusion, the participation of the partners is essential to achieve a solid financial liquidity, allowing them to fulfill all the obligations contracted, the most important factor being the financial knowledge of the partners, this contributes to generating a greater understanding of the financial field and attending to their responsibilities contracted.
\end{abstract}

Keywords: Savings and credit cooperatives, financial education, financial management.

Artículo recibido: 15 enero 2020 Aceptado para publicación: 14 febrero 2020 Correspondencia: juan.portilla@unsaac.edu.pe Conflictos de Interés: Ninguna que declarar 


\section{INTRODUCCIÓN}

Actualmente la sociedad conocimiento, es cada vez más exigente en las diferentes áreas del conocimiento, pero en el estudio se abordará específicamente en el ámbito de las finanzas, específicamente la educación financiera, que viene a ser un factor que no se considera como tal en Latinoamérica, debido a que se observa muchos emprendimientos que quieran y tampoco se evidencia un cultura financiera orienta al ahorro, siendo esta muy relevante porque les da la posibilidad a las personas a administrar coherentemente sus ingresos, ahorros e inversiones; su importancia reside en la promoción de cultura de ahorro e inversión, además de aleccionar la manera eficiente de cómo administrar tales recursos económicos y la manera de como invertirlas prudentemente. (Raccanello \& Herrera, 2014, pp.119-120)

Al realizar un análisis global, es factible mencionar que al menos el $50 \%$ de la población adulta posee una cuenta financiera formal en una entidad bancaria, por lo cual están sujetas a realizar las diversas operaciones y prestaciones que te brindan estas entidades, a diferencia de Latinoamérica y el Caribe donde solo el 39\% de la población recurre a prestaciones financieras y de este porcentaje solo el $8 \%$ lo hace en entidades formales, el resto del porcentaje mencionado acude a entidades no formales como casas de empeño, agiotistas, etc. lo cual en los procesos puede acarrear a un riesgo muy alto y afectar la estabilidad financiera económica de una persona. (Demirguc-Kunt \& Klapper, 2012, pp.1-5)

En la publicación realizada por el Banco Bilbao Vizcaya Argentaria (2019) o más conocido como BBVA, indica que el solamente el $28 \%$ de los peruanos poseen un buen manejo de conocimientos financieros, además de ocupar el sétimo lugar en Latinoamérica, de manera que alcanzar un bienestar personal y financiero es posible mediante un ahorro programado y la toma de decisiones acertadas a partir del manejo adecuado de la información financiera. El Perú a comparación de los demás países latinoamericanos, Uruguay ocupa el primer lugar con un buen nivel de educación financiera en un $45 \%$, mientras que el último lugar es ocupado por Bolivia con un $24 \%$, por tanto, podemos deducir que Perú está próximo a los últimos lugares, de tal forma se evidencia la necesidad de una buena educación financiera, con solo comparar el buen nivel de educación financiera que alcanzan los países europeos de Suecia y Noruega en un $71 \%$. (párr. 1-4) 
Como se ha indicado en los párrafos anteriores, es importante hacer notorio la necesidad de contar con un alto nivel de educación financiera, lo cual permite que las personas cuenten con la preparación adecuada para la toma de decisiones a cerca de su dinero, en cuanto a las inversiones y formas de ahorro de que puede realizar, para alcanzar este el nivel alto es importante consolidar nuestras costumbres financieras, a partir de una cultura de ahorro, de manera que permita a las personar alcanzar sus proyectos personales, profesionales o enfrentar situaciones inesperadas; asimismo es fundamentar realizar una planificación de los recursos, donde es importante planear las necesidades más urgente e importantes de pendiendo de las exigencias de inversión, es decir, si requiere una fuerte inversión, por tanto es necesario elaborar un presupuesto personal, y finalmente es crucial la responsabilidad, porque es oportuno tener en cuenta el hecho de adquirir compromisos financieros, más aún si se trata de las tarjetas de crédito, evitando la libertar de comparar sin control, debiéndose ser la persona más consciente de los gastos que realizan, teniendo en cuenta los intereses y seguros que se irán cobrando a lo largo del tiempo de contrato. (párr. 5-8)

En el contexto descrito precedentemente, la educación financiera de una persona está directamente asociado a la capacidad de control en el sistema bancario y no bancario, de tal forma que no se adeude, ni tampoco gaste más dinero del que recibe, además de tomar conciencia de cada compra que realiza, evitando los gastos sin control o por capricho, consecuentemente podrá disfrutar de mejor manera de su dinero, incluso podrá destinarlo para el cuidado de su salud física y mental. Ante esta situación es que el objetivo del estudio es explicar la incidencia de la educación financiera de socios en la gestión financiera de la Cooperativa de Ahorro y Crédito «Santo Domingo de Guzmán» del Cusco. Su importancia radica en la necesidad de investigar aquellos factores que directamente estén involucrados en la forma que se administran financieramente las cooperativas de ahorro y crédito (COOPAC), quienes actualmente constituyen uno de los vehículos de cooperación social más antiguos del Perú, por ser fundamentales para la inclusión financiera y desarrollo económico del país, con mayor incidencia en los sectores vulnerables y lejanos del Perú. En consecuencia, se pretende dar respuesta a la siguiente interrogante: ¿Cuál es la incidencia de la educación financiera de los socios en el proceso de la gestión financiera de la Cooperativa de Ahorro y Crédito «Santo Domingo de Guzmán» del Cusco? 
Para resolver dicha interrogante se ha recurrido a los escritos de diferentes autores, tales como el «Contexto de la educación financiera en México» escrito por Amezcua, Arroyo, \& Espinosa (2014), en sus hallazgos menciona aquellos aspectos que reflejan la falta de educación financiera, el primero es debido a la escasa participación de los sectores sociales en los productos y servicios que ofrecen las instituciones financieras, el segundo aspecto son los malos hábitos al momento en que se decide utilizar los productos y servicios financieros, y como tercer aspecto es la falta de planeación financiera, habiendo incidido primariamente en este último aspecto, con respecto a las causas por las que las personas realizan un poco uso de los servicios y producto financieros, la desconfianza que les genera, el no saber dónde solicitarlos, la complejidad de los instrumentos, los altos costos que estos tienen y la cantidad de trámites para obtenerlos, donde le deseo de una estabilidad financiera, es necesario contar con un sistema financiero fuerte, a través de leyes y reglas que promuevan la expansión del ahorro, el crédito, la competencia, las inversiones y en general se tenga una mayor educación y cultura financiera. (pp. 23-24)

El estudio «Estructura organizacional y estilos de liderazgo en Cooperativas de Ahorro y Crédito de Pichincha» escrito por Galarza, García, Ballesteros, Cuenca, \& Fernández (2017), indican que la estructura que vienen utilizando las cooperativas es mayormente funcional, debido a la adecuación realizada al nivel de la misión, objetivos estratégicos, por otra parte, los gerentes consideran en cuanto al tamaño de complejidad organizacional debería estar debidamente adecuado al número de actividades, unidades geográficas y departamentos, debiendo responder a los objetivos de acuerdo a una forma de liderazgo que sea funcional con la misión y visión de la cooperativa.

\subsection{Educación financiera}

De acuerdo a la Organización para la Cooperación y el Desarrollo Económicos (2005), la educación financiera es un procedimiento mediante el cual las personas incrementan su entendimiento, conocimientos y habilidades sobre los conceptos, beneficios y riesgos de los productos financieros, lo cual se logra con la aprehensión de información y un asesoramiento objetivo; el poseer tales capacidades brinda la confianza necesaria para tomar decisiones financieras informadas, asumiendo conscientemente las oportunidades conjuntamente con sus riegos. (p.31) 
Para Bansefi (2008), es un procedimiento de desarrollo de actitudes y habilidades, las misma que se logra con el acceso a información puntual y entendible, además del conocimiento de instrumentos elementales para la administración propicia de recursos, lo cual posibilita:

- Tomar decisiones económicas financieras personales y sociales

- Acceder a productos y prestaciones financieras para mejorar su calidad de vida bajo condiciones de certeza.

Gómez (2009), menciona que la Educación Financiera pretende generar un cambio en el actuar de los agentes económicos de manera que optimicen el modo de administrar los recursos financieros. Además, afirma que la educación financiera es la cesión de habilidades, conocimientos y actitudes básicas que permitan a los individuos adoptar correctas prácticas de administración monetaria, específicamente en manejo de ingresos, ahorro, gasto, endeudamiento, e inversión. (p.8)

La CONDUSEF (2011), en el ABC de la educación financiera nos menciona que esta es un conducto para adquirir conocimientos, de manera que nos permita desarrollar habilidades financieras, la misma que aporte a que tomemos decisiones apropiadas y correctas, lo cual produzca la sensación de tranquilidad y bienestar, tanto personal como social.

El economista peruano Trivelli (2009), manifiesta que la educación financiera es generar una permuta de conducta de los agentes económicos, de forma que se desarrollen capacidades respecto a las finanzas personales y optimicen la manera en la que se manejan los recursos monetarios; además menciona que la educación financiera es producir un cambio de comportamiento de los agentes económicos, de tal manera que desarrollen habilidades sobre sus finanzas personales y mejoren la forma en la que administran sus recursos financieros. Asimismo, manifiesta que la educación financiera nos nutre de datos e información relevante para la toma de decisiones financieras, lo cual elevara el nivel de educación y conciencia de las personas en el aspecto financiero, por lo tanto, se demandara productos y prestaciones de primer nivel, lo que conllevara a acrecentar los niveles de ahorro e inversión además del crecimiento de la economía.

La educación financiera puede coadyuvar a aminorar las barreras a la demanda de inclusión financiera; además esta acrecienta el conocimiento con relación a los productos y prestaciones financieras. La educación financiera al referirnos a la 
protección del consumidor financiero se centra en brindar transparencia a los usuarios respecto a sus derechos y obligaciones, punto que es un complemento relevante a la regulación y fiscalización de los mercados financieros y a la intervención del estado en el mercado.

\subsubsection{La educación financiera en el Perú}

De acuerdo con la FEPCMAC (2015), en el Perú la educación financiera se desarrolló evidentemente lo cual fue recocido internacionalmente por varios organismos. Es relevante señalar además que los proyectos de educación financiera a pesar de su corto tiempo de implantación vienen siendo aceptados y se ha extendido, habiendo más personas involucradas y que fortalecen esta capacidad.

En este entender se resalta que nuestro país fue el primero en la región en añadir temas de educación financiera en el Diseño Curricular Nacional en 2009, con lo cual el estado le da la importancia debida a este tema en la etapa escolar.

Los programas de educación financiera implantados mostraron resultados positivos, pero a pesar de ello para garantizar la sostenibilidad y una mejor cobertura a dichos programas es imprescindible empezar a establecer el diseño de una estrategia nacional de educación financiera que englobe a todos los actores y tenga una finalidad y metas concretas.

En este entender, es necesario que los programas de educación financiera busquen crear cambios en el actuar de quienes estén inmersos en esta, con el propósito de mejorar la capacidad de manejar los recursos monetarios financieros. Por tanto, el papel de las instituciones financieras, específicamente en las microfinancieras que se hallan más en contacto con la sociedad es generar impactos positivos en los clientes que recién se incorporan al sistema financiero, lo cual es beneficioso y se evidenciara en una sana cartera de créditos.

\subsubsection{Conocimiento financiero}

La Organización para la Cooperación y el Desarrollo Económico (2005), define a la educación financiera como el procedimiento por el cual los clientes financieros e inversores adquieren una mejor perspectiva y conocimientos respecto a los productos y a los conceptos financieros, lo cual se logra por medio de instrucción, información y consejos objetivos, de manera que se posea un mejor conocimiento respecto a las 
oportunidades y riesgos financieros, además de la toma consciente e informada de decisiones con el objetivo de salvaguardar y mejorar su condición financiera.

En base a la premisa ya mencionada el conocimiento financiero involucra el empleo de estos saberes con la finalidad de mejorar la calidad de vida de las personas, dado que brinda elementos relacionados con la planeación y la administración de los recursos económicos de las familias.

Por su parte el Fondo de las Naciones Unidas para la infancia (2013), menciona que la educación financiera y social, tiene como finalidad imbuir a los niños a ser ciudadanos social y económicamente empoderados, dotándolos de las actitudes y los conocimientos necesarios para ser agentes activos capaces de transformar su comunidad y la sociedad.

\subsubsection{Habilidades financieras}

La habilidad financiera comprende desde el proceso de planificar los gastos y presupuestarlos, hasta lograr financiación; tal habilidad nos brinda la capacidad de invertir coherentemente, emplear razonablemente los recursos monetarios, de tomar decisiones financieras inteligentes, tomando en consideración los riesgos existentes todo con el propósito de cuidar la estabilidad financiera. (Visa Inc., 2015)

La concepción de administrar inteligentemente los recursos monetarios en un inicio puede ser compleja y abrumadora, por tanto, se debe seguir un procedimiento con el cual se busca que el entendimiento de las finanzas personales sea óptimo, lo que conllevara a una mejor administración del dinero. La habilidad financiera nos menciona que el primer paso a dar es evaluar la situación financiera actual y en base a ello plantearse objetivos para mejorarla, además de seguir adquiriendo conocimientos; con lo cual la persona podrá manejar de mejor manera sus recursos monetarios financieros. (Visa Inc., 2015)

\subsubsection{Actitudes financieras}

La Comisión Nacional del Mercado de Valores, Banco de España y Servicio de Formación (2004), menciona que las actitudes financieras dan a entender todos los aspectos conexos que el dinero y las finanzas tienen con los aspectos psicológicos; las decisiones financieras no solamente se fundamentan en la razón, el conocimiento y la lógica, sino que se influencias por emociones, valores personales y la presión social.

Este componente emocional suele presentarse con más intensidad en personas que poseen una pobre educación financiera, quienes se dejan influenciar por sus aspectos 
psicológicos al momento de gastar o invertir; por el contrario, una persona con mayor cultura, educación y conocimiento financiero no da lugar a que sus emociones intervengan al momento de realizar sus actividades relacionadas con su gasto o inversión dineraria. (p.2)

La economía conductual analiza y estudia el comportamiento de las personas respecto a recursos monetarios considerando la psicología. Reconoce que las personas somo seres altamente emocionales, en ocasiones irracionales; por tanto, da a entender que al momento de tomar decisiones financieras no solo se consideran aspectos lógicos, racionales y de análisis, sino que también aspectos emocionales, de heurística y prejuicios. (p.3)

Poseer mayores recursos dinerarios involucra poseer más oportunidades, brinda las facilidades para realizar las acciones que uno desee, contar con una mejor calidad de vida; para lo cual no es indispensable ser millonario, esto se puede lograr con una buena planeación, constancia y ahorro, con lo cual se puede pretender a poseer una seguridad financiera necesaria para no atravesar por dificultades. El dinero no involucra que las personas que la poseen en cantidad con relación a las que no sean mejores, pero si hace notoria de que aquellas que la tienen en cantidad accedan a mayores opciones en diversos ámbitos. (p.5)

\subsubsection{Bienestar financiero}

León (2006), afirma que el bienestar financiero está vinculada a la situación financiera de una persona, familia u comunidad, la misma que se fundamenta en cualidades percibidas y cualidades objetivas las mismas que se califican contra estándares de comparación a cualidades evaluadas de tal situación financiera. Además, plantea que el bienestar financiero es una función de características individuales, de comportamientos financieros y de acontecimientos financieros estresantes.

Prawitz, et al. (2007), menciona que, en los últimos años, algunos estudios demostraron fehacientemente vínculos entre el estrés financiero y la salud; se muestra que aquellas personas que experimentan un grado elevado de angustia financiera presentan un nivel elevado de estrés lo cual afecta de alguna manera en su salud. Para evitar llegar a este punto es relevante la adquisición y la asimilación de conceptos relacionados con la educación financiera. 
El bienestar financiero está sujeta a medidas subjetivas y objetivas de la situación financiera, además de la percepción personal respecto a atributos objetivos de la situación financiera, posterior a la comparación de este contra ciertos estándares. Los estándares de comparación contienen horizontes de tiempo propias que conciernen a las variables de puntos de referencia empelados en estudios previas de bienestar financiero. Los atributos objetivos son definidos como indicadores cuantitativos de la situación financiera, como el ingreso y el tamaño de la familia.

\subsection{Gestión financiera}

De acuerdo con Terrazas (2009), la gestión financiera es una actividad que se ejecuta en una entidad, se hace cargo de la planificación, organización, dirección, control, monitoreo y coordinación respecto a los recursos financieros disponibles, con el objetivo de crear más beneficios o resultados. El propósito radica en que la entidad opere efectivamente, contribuir a la efectiva toma de decisiones financieras, y propiciar oportunidades adecuadas de inversión.

Para la correcta operación de una organización es relevante una excelente administración financiera. Sin embargo, existen diferencias en las metas organizacionales de los gerentes financieros, en una entidad comercial el objetivo primordial radica en maximizar las utilidades, en cambien en una entidad de prestaciones con carácter social su objetivo radica en el empleo de los recursos para obtener el bienestar común. El gerente financiero de una entidad tiene como fin primordial conseguir incrementar la relación costo - beneficio por medio de acciones diarias; lo que involucra considerar aspectos del correcto manejo en la política crediticia, de inventarios, compras, etc. las mismas que deben tomarse acertadamente y con el mínimo de riesgo posible, en el corto, mediano y largo plazo; en este contexto la precisión, administración y sistematización de la información financiera es primordial para la consecución de un objetivo. Una labor relevante del gerente financiero es también velar por los demás factores esenciales como lo son la administración de la infraestructura, del recurso humano, de las operaciones y formas administrativas. Por lo cual es relevante establecer la conexión sistémica de tales factores, elementos para contribuir al proceso de toma de decisiones organizacional. 
Para Martínez (2016), la gestión financiera hace referencia al proceso de toma de decisiones y análisis de datos con el fin de mejorar la administración y la utilización de los recursos financieros de una organización para la consecución de sus objetivos.

En la actualidad, la gestión financiera esta conexa al concepto de valor empresarial, por lo que la gestión financiera forma parte del proceso, y se enfoca en el uso efectivo y eficiente de los recursos propios, de los compromisos financieros y demás fondos; así como de la correcta toma de decisiones por tanto la maximización de beneficios y del valor de la organización.

Para la presencia de una gestión financiera de carácter previa, se debe establecer objetivos organizacionales, además de diseñarse una apropiada estructura y aplicarla. Los objetivos organizacionales pueden ser diversos, pueden ser de naturaleza financiera, económica, estratégica, etc. la cual depende de los objetivos planteados.

Para Pinar \& Rivas (2002), se denomina gestión financiera (o gestión de movimiento de fondos) a los diversos procedimientos que se centran en obtener, mantener y empelar recursos monetarios en sus diversos modos de empleo. La gestión financiera transforma la misión y visión en operaciones monetarias.

De acuerdo con el documento del grupo del Banco Mundial, Dirección Sub regional para América Latina, la gestión financiera se centra en la planeación, ejecución y control de los recursos financieros de una organización, además engloba los sistemas conexos a estos recursos, como también a los procesos direccionados a la consecución de objetivos de la organización. Se centra en el uso de fondos y su rendición por tal acción.

\section{Entre los sistemas que la integran mencionamos:}

- Sistemas de planificación

- Sistema de flujo de fondos

- Sistemas de información

- Sistemas de control

La gestión financiera es empleada para la administración de recursos financieros de los proyectos la apropiada atención a la economía y la eficiente utilización de tales recursos.

- Coadyuva a la consecución de los objetivos de un proyecto, proporcionando información valiosa para mejorar el proceso de toma de decisiones. 
- Indica además el empleo correcto de los fondos destinados a un proyecto.

\subsubsection{Planificación financiera}

Gitman (2007), menciona que la planeación financiera funda la forma de consecución de los objetivos, por otro lado, representa también el sustento de toda la actividad económica de la organización. Es importante también recalcar que además procura la previsión de los requerimientos venideros de forma que las actuales sean satisfechas según con el objetivo fijado en las actividades de la organización.

La planeación financiera engloba una serie de instrumentos, método y propósitos, con la finalidad de instaurar en una organización, predicciones y fines económicas financieras por lograr, considerando los recursos con los que se cuentan y los que harán falta para conseguirlo. La planeación financiera establece la dirección que debe perseguir la organización para lograr la consecución de sus objetivos estratégicos, tiene como finalidad acrecentar la rentabilidad, fijar la cuantía apropiada de efectivo como de las fuentes de financiamiento, además de establecer el grado de ventas y concertar los egresos que conciernen al nivel de operaciones, etc. La planeación financiera incide en las actividades de la organización ya que facilita rumbos que guían, coordinan y controlan las actividades para la consecución de las metas organizacionales. (p.102)

Existen dos factores claves del procedimiento de planificación financiera y son: a) La planificación de efectivo y b) La planificación de utilidades. La primera involucra la realización del presupuesto de caja de la organización; y la segunda comprende la realización de estados proformas. Tanto el presupuesto de caja como los estados proforma son útiles para la planeación financiera interna, además los financistas lo solicitan y demandan.

De Urquijo (1977), afirma que la planeación financiera y los procesos de control se hallan conexas, debido a que se requiere analizar los resultados de la planeación financiera. La planeación y el control involucran el uso de proyecciones que consideran como base las normas y el desempeño de un procedimiento de retroalimentación y de ajuste para acrecentar el rendimiento. El proceso de planeación y control financiero involucra la gestación de pronósticos y el empleo de diversos tipos de presupuestos. Se deben desarrollar sistemas de presupuestos para todos y cada una de las unidades importantes de la organización. Para que haya un excelente plan financiero es indispensable que exista un excelente plan general para la organización. Por tanto, la 
calidad de los programas, planes y presupuestos financieros se halla condicionada a los distintos componentes de la planificación organizacional.

\subsubsection{Organización financiera}

Para International Business Machines (2008), la organización financiera proporciona una eficiencia superior al momento de coadyuvar la integración de la información en toda la organización es una de los factores esenciales y diferenciadores para las organizaciones y de esta manera obtener resultados óptimos.

La organización financiera es la unidad parte de la administración que se enfoca en decisiones de inversión, manejo dinerario, fuentes de financiación, administración de activos, control de costos y maximización de beneficios o utilidades.

La organización financiara se apoya en los procesos administrativos y los registros contables, requiriendo datos verídicos y fiables para la toma de decisiones gerenciales. La contabilidad documenta y registra monetariamente todas las actividades de una organización, ya sean ventas, adquisiciones, contrataciones, efectivo, inventarios, cuentas por cobrar, deudas, activos fijos y patrimonio; las cuentas mencionadas son registradas en la contabilidad y se presentan a la gerencia financiera a través de los estados financieros, siendo las primordiales el balance general y el estado de resultados.

\subsubsection{Dirección financiera}

Aragó \& Cabedo (2011), mencionan que el propósito de la dirección financiera dentro de una entidad es la de acrecentar el valor de la organización; desde la perspectiva de los accionistas las decisiones financieras que incrementen el valor de la empresa se asumen como correcta.

La dirección financiera debe hallarse bajo el manejo de personas que cuenten con varias habilidades y competencias, como un conocimiento amplio y profundo del comportamiento de los mercados financieros, debido a que es el sector de donde se puede obtener los datos necesarios para que los planes de inversión sean seguros y beneficiosos para la organización.

La dirección financiera es una división esencial en el procedimiento de toma de decisiones relevantes dentro de una entidad, la dirección financiera además se hace cargo de que las decisiones que se tomen puedan ser asumidas con criterios drásticos, buscando el bienestar financiero de la organización. 


\subsubsection{Control financiero}

De acuerdo con Amat (2016), los sistemas de control financiero existen desde la era de las civilizaciones antiguas, la misma que evoluciono, y gracias a la innovación se desarrolló y mejoró hasta la época actual. El control financiero es una acción relevante del área financiera de una organización, debido a que se emplea para controlar cuentas, además de tener conocimiento respecto a los gastos y la procedencia de los ingresos. Esta además de contribuir con la información relacionada a la propia organización, constituye también el sustento de las diversas acciones o permutas financieras que se desee ejecutar, por lo que esta es un punto de referencia esencial para conservar un correcto equilibrio presupuestario.

El control financiero es una actividad que se centra en la supervisión y vigilancia del estado financiero de una organización, lo que pretende es que no se presente una situación de déficit entre los ingresos y egresos. Es una actividad esencial debido a que de esta depende el saneamiento de cuentas, además de que posibilita conocer el estado de cuentas y ubicar los probables agujeros financieros, y poder tomar acciones frente a ello.

\section{MATERIALES Y MÉTODOS}

Para el procesamiento y análisis estadísticos respectivo se tienen datos transversales de los socios de la Cooperativa de Ahorro y Crédito «Santo Domingo de Guzmán» del Cusco, cuyas edades fluctúan entre 22 a 75 años, donde la mayoría de los socios fluctúa entre las edades de 30 a 40 años, que en promedio es 41,08 años, es decir una población adulta, con una desviación estándar de 11,150 años, quienes difieren entre edades en aproximadamente 11 años con respecto a la media, de tal forma la unidad de estudio, que estuvo caracterizada de acuerdo a lo mencionado, asciende a un total de 51961 socios activos. La muestra de origen en su mayoría de aquellos socios que se encuentran en la provincia del Cusco, lugar donde mayormente residen los socios de la Cooperativa de Ahorro y Crédito «Santo Domingo de Guzmán», fueron seleccionados mediante un muestreo probabilístico sistemático, de cuyo cálculo se tiene un total de 245 socios, de manera que todos los participantes del estudio tenían como mínimo dos años de participación continua hasta la fecha de recogida de los datos. Los instrumentos de recolección de información utilizados corresponden a los cuestionarios, quienes cuentan con la fiabilidad del caso, por una parte, la validez por juicio de expertos, en más de 
$80 \%$ respecto de contenido, estructura y forma, y el análisis de la consistencia interna mediante Alfa de Cronbach con un valor del 0,926 en el caso del primer cuestionario, y el segundo con un índice del 0,941; los cuestionarios de educación y gestión financiera fueron aplicados a los socios antes caracterizados y en la cantidad seleccionada, adicionalmente se realizó el análisis de las colocaciones de créditos, captaciones mediante las cuentas de ahorro de los socios y la rentabilidad que se tiene como parte de la gestión financiera de la cooperativa. Para obtener los datos se optó por solicitar una autorización a la cooperativa, así como también la información financiera más relevante de los socios activos para realizar el análisis estadístico respectivo. (Bernal, 2010, p.250)

El diseño de la investigación seleccionado para la presentación de la información es de acuerdo con la clasificación realizada por Hernández, Fernández, \& Baptista (2014), porque el tipo de estudio fue el explicativo, porque se fundamenta en la búsqueda de los hechos que determinan la ocurrencia de la gestión financiera, es decir, como afecta la educación financiera de los socios a la gestión financiera de la cooperativa. Con relación a lo mencionado el diseño es el transversal correlacional causal, pero no existiendo ninguna manipulación o intervención por parte del investigador, por tanto, el diseño es no experimental.

Las mediciones realizadas a las variables educación y gestión financiera, fueron analizadas mediante la estadística descriptiva, a través de los diagramas de cajas y bigotes (media y desviación estándar) sementados por género, todos los datos corresponden a las respuestas de los socios, las cuales fueron procesadas de acuerdo a la sumatoria por dimensiones y por el total de las variables, posteriormente se realizó el análisis estadístico inferencial, mediante el estadígrafo correlación lineal de Pearson y la regresión lineal que responden al análisis bivariado y multivariado respectivamente, de datos primarios obtenidos de los cuestionarios. (Triola, 2004)

Respecto a la medición de la variable se consideró el siguiente cuadro de operacionalización de variables para generalizar los conceptos respecto a la educación y gestión financiera. 


\begin{tabular}{|c|c|}
\hline Variables/Dimensiones & Indicadores \\
\hline \multicolumn{2}{|l|}{ Variable educación financiera } \\
\hline Dimensión conocimiento financiero & $\begin{array}{l}\text { - Costo - Beneficio de los productos y servicios } \\
\text { financieros. } \\
\text { - Gestión en los servicios financieros. } \\
\text { - Riesgos financieros. } \\
\text { - Derechos y obligaciones de los socios. }\end{array}$ \\
\hline Dimensión habilidades financieras & $\begin{array}{l}\text { - Manejo de alternativas crediticias. } \\
\text { - Decisión de ahorro e inversiones. } \\
\text { - Rentabilidad de los ahorros e inversiones. } \\
\text { - Preparación para situaciones imprevisibles. }\end{array}$ \\
\hline Dimensión actitudes financieras & $\begin{array}{l}\text { - Autocontrol } \\
\text { - Toma de decisiones } \\
\text { - Relación con el sistema financiero }\end{array}$ \\
\hline Dimensión bienestar financiero & $\begin{array}{l}\text { - Administración de finanzas personales. } \\
\text { - Elaboración plan financiero } \\
\text { - Oportunidades y riesgos financieros }\end{array}$ \\
\hline \multicolumn{2}{|l|}{ Variable gestión financiera } \\
\hline Dimensión planificación financiera & $\begin{array}{l}\text { - Planes estratégicos. } \\
\text { - Sistema de gestión contable adaptado. }\end{array}$ \\
\hline Dimensión organización financiera & $\begin{array}{l}\text { - Análisis financiero. } \\
\text { - Presupuesto anual. } \\
\text { - Asignación de recursos de acuerdo con las } \\
\text { necesidades. } \\
\text { - Organización de las colocaciones de créditos y } \\
\text { captaciones por ahorros }\end{array}$ \\
\hline Dimensión dirección financiera & $\begin{array}{l}\text { - Gestión del recurso humano. } \\
\text { - Capacitación de directivos y personal. } \\
\text { - Seguimiento de las colocaciones de créditos y } \\
\text { captaciones. }\end{array}$ \\
\hline Dimensión control financiero & $\begin{array}{l}\text { - Evaluación de metas y objetivos } \\
\text { - Resultados de las colocaciones de créditos y } \\
\text { captaciones } \\
\text { - Efectividad de los planes estratégicos }\end{array}$ \\
\hline
\end{tabular}

Fuente: Adaptación realizada del Plan Nacional de Educación Financiera publicado por el Banco de La Nación (2017) y lo mencionado por Kotler (2003) y Álvarez (2017) respecto a la gestión financiera. 


\section{RESULTADOS Y DISCUSION}

\subsection{Resultados}

Los datos obtenidos fueron obtenidos de un total de 245 socios, caracterizados principalmente por ser activos o con participación continua por lo menos en 2 años consecutivos, el cual fue recolectado entre los meses octubre a diciembre del año 2019.

Tabla 1: Estadísticos descriptivos de la variable educación financiera y sus dimensiones

\begin{tabular}{lcccc}
\hline \hline Dimensiones/Variable & $\mathbf{n}$ & Media & $\begin{array}{c}\text { Desviación } \\
\text { estándar }\end{array}$ & Varianza \\
\hline Conocimiento financiero & 245 & 52,27 & 10,286 & 105,802 \\
Habilidades financieras & 245 & 10,33 & 3,093 & 9,565 \\
Actitudes financieras & 245 & 10,01 & 2,653 & 7,041 \\
Bienestar financiero & 245 & 12,18 & 3,412 & 11,640 \\
Educación financiera & 245 & 84,78 & 17,854 & 318,754 \\
\hline \hline
\end{tabular}

Fuente: Elaboración en base a resultados del instrumento.

De acuerdo con lo mencionado por el Plan Nacional de Educación Financiera publicado por el Banco de La Nación (2017), menciona:

«Lograr que todas las personas mejoren su bienestar financiero a través de la administración eficiente de su dinero» (p.4)

En ese entender los resultados de la tabla 1, muestra los criterios por tener en cuenta para que las personas alcancen el bienestar financiero que desean alcanzar, a través de un adecuado conocimiento financiero $(\mathrm{DE}=10,286)$ de los productos y servicios financieros (Costo-beneficio), el cual muestra la mayor dispersión de datos respecto a la media, con relación a las respuestas de los socios de la cooperativa, lo cual indica que no todos los socios manejan un conocimiento adecuado respecto a los servicios, riesgos y obligaciones financieras, evidenciándose en algunos casos un desconocimiento completo; en el caso de las habilidades financieras ( $\mathrm{DE}=3,093)$, esto demuestra que existe un mejor manejo de alternativas crediticias, se apoyan de ahorros e inversiones sino no estarían como socios de la cooperativa- para obtener mejor rentabilidad y enfrentar situaciones imprevisibles; respecto a las actitudes financieras $(\mathrm{DE}=2,653)$, la desviación estándar indica la menor dispersión entre las demás variables, de manera que existe un mejor autocontrol, toma de decisiones y se relacionan con el sistema 
financiero de la cooperativa, es decir, que si son socios activos, es decir, que buscan estar informados respecto a los dineros que han venido aportando a lo largo del tiempo; con relación al bienestar financiero $(\mathrm{DE}=3,412)$, se evidencia una mejor administración de las finanzas personales, porque los estadísticos descriptivos, demuestran que no hay demasiada dispersión, pero también aún falta incidir en la administración personal de sus finanzas, tampoco demuestran la elaboración de un sólido plan financiero para evitar los riesgos y orientarse a las oportunidades financieras con el fin de afrontar las dificultades financieras. En general la educación financiera muestra una considerable desviación estándar $(\mathrm{DE}=17,854)$, de tal forma es evidencia que cada dimensión o criterio sobre todo el conocimiento y actitudes no es adecuadamente manejado por los socios de la cooperativa, para alcanzar el bienestar financiero, pero es importante resaltar que existen habilidades, pero con un mejor conocimiento y actitud beneficiará a todos los socios de la cooperativa.

Tabla 2: Estadísticos descriptivos de la variable gestión financiera y sus dimensiones

\begin{tabular}{lcccc}
\hline \hline Dimensiones/Variable & $\mathbf{n}$ & Media & $\begin{array}{c}\text { Desviación } \\
\text { estándar }\end{array}$ & Varianza \\
\hline Planificación financiera & 245 & 22,66 & 5,510 & 30,364 \\
Organización financiera & 245 & 26,76 & 6,617 & 43,788 \\
Dirección financiera & 245 & 20,77 & 5,176 & 26,786 \\
Control financiero & 245 & 18,94 & 4,781 & 22,861 \\
Gestión financiera & 245 & 89,13 & 19,849 & 393,999 \\
\hline \hline
\end{tabular}

Fuente: Elaboración en base a resultados del instrumento.

Álvarez (2017), en sociedad del conocimiento y el desarrollo tecnológico, como es el avance de la comunicación, donde el fenómeno de la innovación financiera propone el surgimiento de nuevos productos financieros, señala que:

«...la velocidad de los cambios ocurridos en el mercado impacta significativamente en las organizaciones, las cuales requieren adaptar, tanto sus recursos como formas de trabajo». (p. 339)

La tendencia de la globalización, durante años no fue debidamente considerada en los países latinoamericanos con respecto al sistema financiero, en este contexto la definición dada por Kotler (2003) citado por Álvarez (2017) explica que: 
«...la gestión financiera es aquella que se compone de ingresos, gastos, activos y pasivos que se llevan comúnmente en las oficinas generales de una organización que proporciona una serie de puntos de atención bien definidos para la aplicación y el mantenimiento de los controles presupuestarios y de tipo operacionales». (p.341)

Los resultados producto de la percepción que tienen los socios de la cooperativa, muestran que la gestión financiera presenta una notoria dispersión en cuanto a la planificación financiera, dado por una desviación estándar $(\mathrm{DE}=5,510)$ determinando la existencia de planes estratégicos y del sistema de gestión contable poco adaptados a las necesidades de los socios, debido a que no evidencia una percepción favorable en la mayoría de los socios de la cooperativa, respecto a la organización financiera ( $\mathrm{DE}=6,617)$, esta demuestra que se requiere una forma más comprensible del informe de los análisis financieros, asignación de recursos y tener una mejor organización de las colocaciones y captaciones por parte de la cooperativa, en el caso de la dirección financiera $(\mathrm{DE}=5,176)$ se observa que todavía falta una mejor capacitación del personal que mantiene constante comunicación con los socios, y también es necesario incidir en formular un adecuado seguimiento de las colocaciones de créditos y captaciones, debido a que se observa dispersión de las respuestas dadas por los socios, finalmente en el control financiero $(\mathrm{DE}=4,781)$ también muestra una dispersión ligeramente menor al resto de las dimensiones, lo cual indica que la evaluación de metas y objetivos, no está al alcance de todos los socios, y mayor razón si son socios activos, es importante también que se publiquen en porcentajes respecto al número de colocaciones de créditos y captaciones de forma anual, como mínimo, para que los socios puedan sentirse más seguros respecto a los ahorros contraídos con la cooperativa y de ser el caso en alguna inversión.

Una percepción general de la gestión financiera de acuerdo a lo mencionado por Kotler (2003) citado por Álvarez (2017), se evidencia que la cooperativa se esmera por alcanzar una adecuada planificación, organización, dirección y control financiero, de los ingresos, gastos, activos y pasivos de la cooperativa, teniendo en cuenta un balance general y por cada agencia, para evaluar la eficacia y eficiencia en la atención de los socios, así como la seguridad que deben ofrecerles, para fidelizarlos, y generar solidez financiera, pero como se evidencia la dispersión en sus respuestas, se observa que la gestión financiera no es sólida en cuanto a la percepción de los socios. 
Figura 1. Diagrama de cajas y bigotes de la educación financiera por nivel de estudios de los socios de la cooperativa

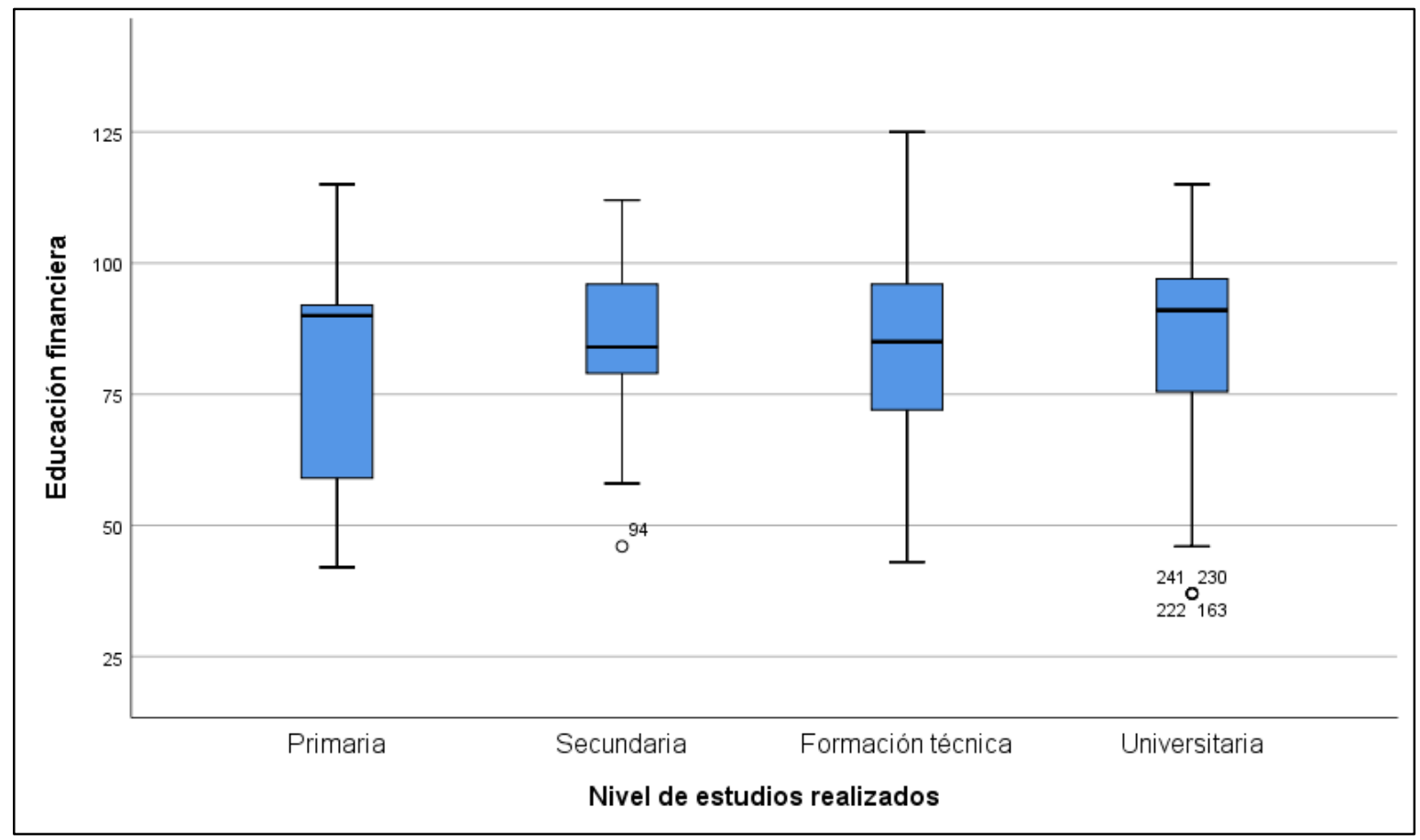

Fuente: Elaboración propia en base a los resultados en IBM SPSS.

El diagrama de cajas y bigotes evidencia que la mayoría de los socios que tienen estudios de primaria presentan dificultades en la educación financiera, observándose una mayor dispersión, de tal forma, este grupo muestra un menor conocimiento, habilidades, actitudes y consecuentemente menor bienestar financiero, a diferencia de los demás niveles de estudios de los socios, tales como secundaria, formación técnica y universitaria, que en cierta forma este problema ocurre en menor proporción en estos niveles de educación, cabe destacar que el nivel de estudios de formación técnica que tienen los socios, presentan algunos problemas significativos en cuanto al conocimiento financiero, en cuanto al costo beneficio de los productos y servicios financieros, pero la mayoría de los socios en las respuesta individuales, demuestra tener un regular manejo de la administración de finanzas personales y tampoco han elaborado un plan financiero de acuerdo a sus objetivos y necesidades. 
Figura 2. Diagrama de cajas y bigotes de la gestión financiera por nivel de estudios de los socios de la cooperativa

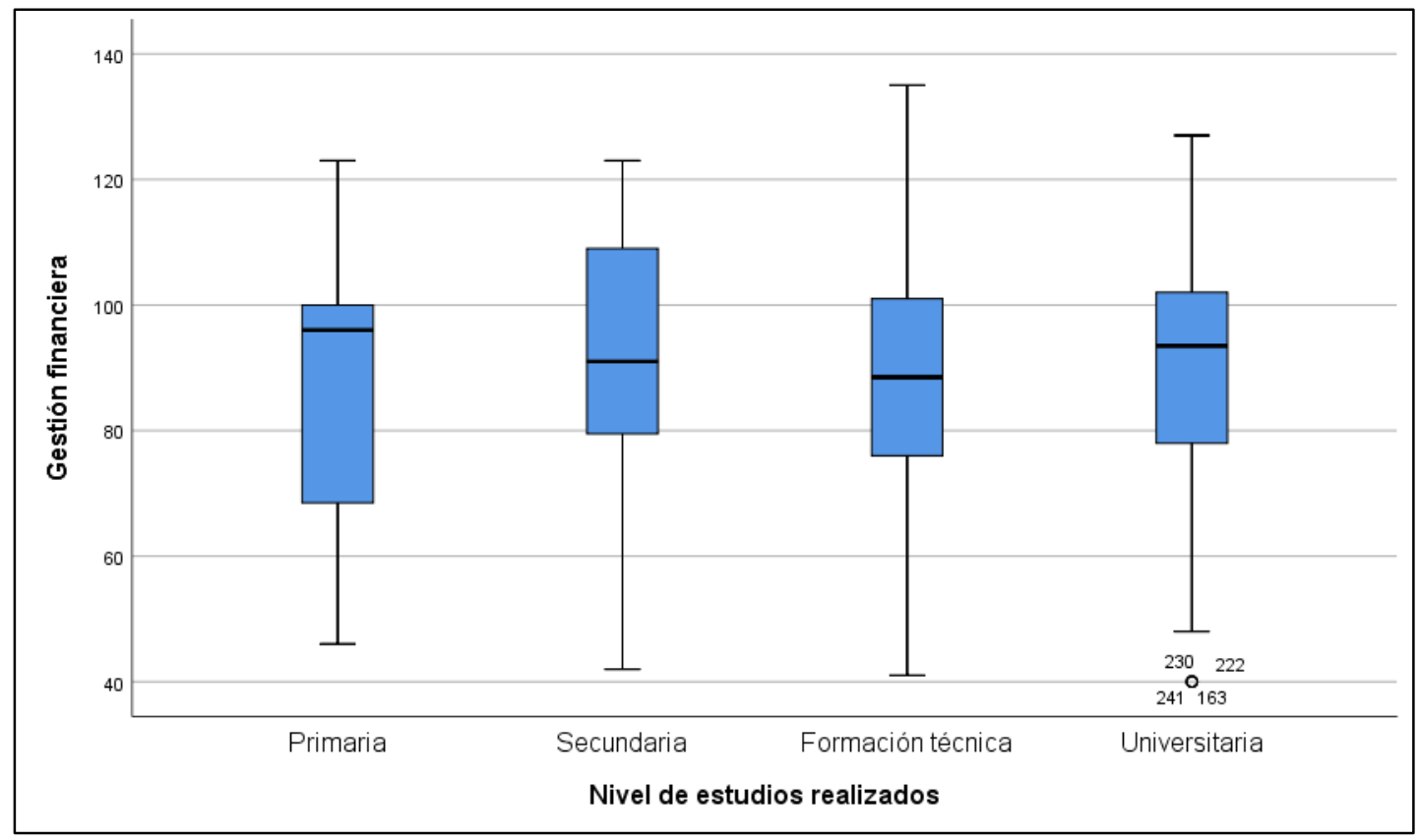

Fuente: Elaboración propia en base a los resultados en IBM SPSS.

La gestión financiera que viene desarrollando la cooperativa, de acuerdo con el nivel de estudios de los socios activos, se observa en el nivel de estudios de primaria, son aquellos que perciben un baja capacidad en cuanto a la planificación, organización, dirección y control financiero que viene desarrollando la cooperativa, esto podría deberse a que las personas con un nivel de estudios de primaria, podrían no comprender adecuadamente las acciones que viene desarrollando la cooperativa, por ejemplo, comprende un plan estratégico y un sistema de gestión de contable requieren el manejo de la tecnología y mayor capacidad de comprensión, tampoco se puede menospreciar a estos socios, porque existen autodidactas muy bien preparados, pero los casos que alcanzan de acuerdo al conocimiento empírico, son muy pocos, en tal sentido se evidencia que los socios con un mejor nivel de estudios, caso secundario, técnico y universitario indican que la cooperativa demuestra un manejo en cierta medida bueno, porque cumple con sus expectativas, pero tampoco se puede evidenciar rasgos muy llamativos en cuanto a la forma que viene realizando la gestión financiera en la cooperativa de ahorro y crédito «Santo Domingo de Guzmán» del Cusco, para evitar esta situación las cooperativas cuentan estructuralmente con un comité de educación, quien debería encargarse de fomentar la educación financiera de los socios, además de involucrarlos más con los objetivos y metas de la cooperativa. 
Tabla 3: Estadísticos bivariados entre las variables educación y gestión financiera

\begin{tabular}{llccc}
\hline \hline Variables & Educación financiera & $\begin{array}{c}\text { Correlación } \\
\text { de Pearson }\end{array}$ & $\begin{array}{c}\text { Sig. } \\
\text { (bilateral) }\end{array}$ & n \\
\hline $\begin{array}{l}\text { Educación } \\
\text { financiera }\end{array}$ & Gestión financiera & 1 & & 245 \\
\hline $\begin{array}{l}\text { Gestión } \\
\text { financiera }\end{array}$ & Educación financiera & $0,899^{* *}$ & 0,000 & 245 \\
\hline \hline
\end{tabular}

Fuente: Elaboración en base a resultados del instrumento.

**. La correlación es significativa en el nivel 0,01 (bilateral).

Realizando el análisis del estadígrafo correlación lineal de Pearson, mediante el método de la lectura del valor P mencionado por Triola (2004), consiste específicamente en comparar el nivel de significancia con el valor $\mathrm{P}$ obtenido por el programa estadístico para aceptar la hipótesis alterna, realizando esta operación podemos observar que el valor $\mathrm{P}=0,000$ menor al nivel de significancia del 5\%=0,05 de tal manera se acepta la hipótesis alterna, demostrándose la existencia de la correlación, además de encontrarse una buena o alta correlación entre las variables educación y gestión financiera, dado por el valor de $\ll \mathrm{r}=0,899 »$ por su valor positivo es una correlación directa.

Figura 3. Diagrama de dispersión de puntos de las respuestas de los socios respecto a la educación con la gestión financiera

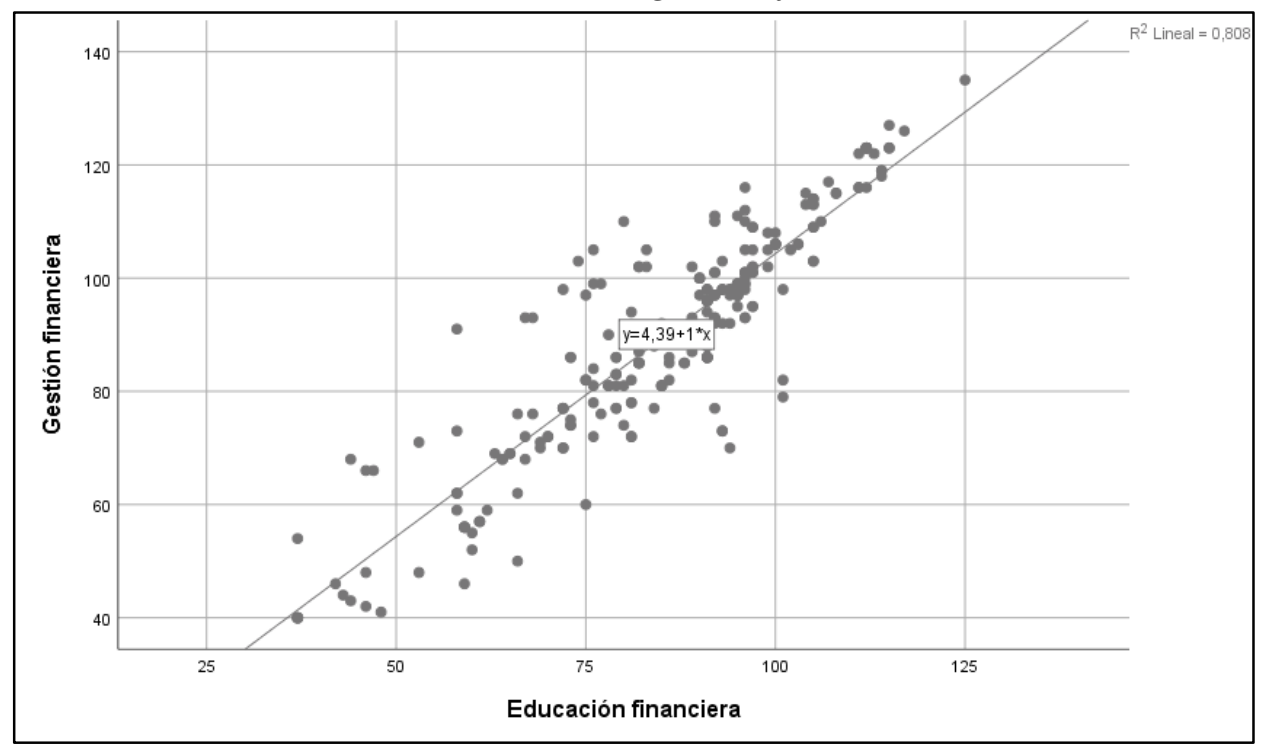

Fuente: Elaboración propia en base a los resultados en IBM SPSS.

El diagrama de puntos, muestra la existencia de aglutinamiento de los puntos o las respuestas de los socios con respecto a la educación y gestión financiera, lo cual 
demuestra la existencia de la correlación entre estas dos variables, además se puede observar que la recta de ajuste presenta una pendiente positiva, por tanto, mientras mejor sea la educación financiera de los socios, la cooperativa presentará un mayor progreso de la gestión financiera, logrando en el tiempo una alta solidez financiera en el mercado y también cumplirá con los objetivos y metas formulados en su plan estratégico.

Tabla 4: Correlaciones entre las dimensiones de la variable independiente educación financiera con la variable dependiente gestión financiera

\begin{tabular}{lccc}
\hline \hline Gestión financiera* & $\begin{array}{c}\text { Correlación de } \\
\text { Pearson }\end{array}$ & valor $\boldsymbol{P}$ & $\mathbf{n}$ \\
\hline Conocimiento financiero** & 0,910 & 0,000 & 245 \\
Habilidades financieras** & 0,637 & 0,000 & 245 \\
Actitudes financieras** & 0,763 & 0,000 & 245 \\
Bienestar financiero** & 0,789 & 0,000 & 245 \\
\hline
\end{tabular}

Fuente: Elaboración en base a resultados del instrumento.

* Variable dependiente

** Dimensiones de la variable independiente

El análisis de la correlación lineal de Pearson entre las dimensiones de la variable independiente educación financiera con la variable dependiente gestión financiera, se observa que existe la correlación debido a que el valor $P=0,000$ es menor al nivel de significancia del $5 \%=0,05$; pero existe una diferencia considerable en cuanto al coeficiente de correlación entre el conocimiento y la gestión financiera, debido a que alcanza un 0,910 muy próximo a la unidad, lo cual indica una alta correlación, mientras que en los demás casos tales como las habilidades, actitudes y bienestar financiero muestra una buena correlación con la gestión financiera.

Es posible indicar que, en alguna medida a través de la mejoría continua del conocimiento, habilidades, actitudes y el bienestar financiero que vayan desarrollando los socios de la cooperativa, impulsará en la mejora de la gestión financiera, inyectando liquidez financiera, lo cual permitirá hacer frente las obligaciones de la cooperativa a corto plazo.

Tabla 5: Estimación de parámetros de la regresión lineal entre las variables educación y gestión financiera 


\begin{tabular}{lcccc}
\hline \hline \multirow{2}{*}{ Modelo } & $\begin{array}{c}\text { Coeficientes no } \\
\text { estandarizados }\end{array}$ & $\begin{array}{c}\text { Coeficientes } \\
\text { estandarizados }\end{array}$ & t & Sig. \\
\cline { 2 - 4 } & $\mathrm{B}$ & Beta & & \\
\hline $\begin{array}{l}\text { (Constante) } \\
\begin{array}{l}\text { Conocimiento } \\
\text { financiero }\end{array}\end{array}$ & $-10,771$ & & $-12,502$ & 0,000 \\
$\begin{array}{l}\text { Habilidades } \\
\text { financieras }\end{array}$ & 1,867 & 0,968 & 61,928 & 0,000 \\
$\begin{array}{l}\text { Actitudes financieras } \\
\text { Bienestar financiero }\end{array}$ & 0,079 & 0,012 & 1,077 & 0,282 \\
\hline \hline
\end{tabular}

a. Variable dependiente: Gestión financiera

Fuente: Elaboración en base a resultados del instrumento.

Como se puede observar el modelo de regresión lineal indica que las dimensiones en conjunto no forman parte de un modelo predictivo, capaz de determinar a través de estas dimensiones la mejor continua de la gestión financiera, con la necesidad de asegurar la liquidez financiera esperada para atender en el corto plazo a las demandas de los socios de la cooperativa, la única dimensión que forma parte del modelo predictivo para lograr una alta gestión financiera es el conocimiento financiero, estimando que mientras mayor sea en los socios, determina una mejor capacidad de respuesta de la cooperativa con respecto a su gestión financiera, donde el modelo de regresión lineal es: $Y=-10,771+1,867(X)$, donde «Y» viene a ser la variable dependiente gestión financiera $\mathrm{y} \ll \mathrm{X} »$ la variable independiente educación financiera.

\subsection{Discusión}

Los hallazgos del estudio hicieron posible 'explicar la incidencia de la educación financiera de socios en la gestión financiera de la Cooperativa de Ahorro y Crédito «Santo Domingo de Guzmán» del Cusco', dado por el análisis bivariado y multivariado, es decir, a través de los resultados de la correlación lineal de Pearson y la regresión lineal múltiple, se ha observado el comportamiento de las variables, determinando la importancia de la educación financiera en el proceso de la gestión financiera de la cooperativa.

En cuanto a un análisis específico de las colocaciones de créditos que ha venido experimentado la cooperativa en los últimos cinco años demuestra que para el año 2013, en su saldo histórico de cartera por tipo de crédito, se observa a un 67,3\% de participación, a diferencia de los demás tipos de crédito tales como: Hipotecario, microempresa, pequeña empresa en conjunto representan solamente el 32,7\%, por tanto, 
la mayoría de los casos de crédito recae en el consumo del socio activo, realizando dicho análisis para el año 2017, aún se mantiene esta tendencia en un 65\% el crédito por consumo, a diferencia de los otros tipos de consumo que también solamente alcanzan el $35 \%$ durante el periodo de 5 años, confirmando la participación en su mayoría por parte de los socios.

Respecto a las captaciones (ahorros) realizado por la cooperativa, los ingresos financieros a lo largo de los años, por ejemplo, del 2012 con un ingreso de 19965 al 2016 se tuvo un 33520 en miles de soles, se evidencia una diferencia positiva de 13555 expresado en miles de soles, donde el margen financiero bruto alcanza los 20199 en miles de soles, mientras los gastos financieros alcanza los 13321 en miles de soles, observándose que la Cooperativa de Ahorro y Crédito «Santo Domingo de Guzmán» del Cusco. De los créditos otorgados por la cooperativa, se observa que existen ingresos significativos, así como también el aporte pecuniario mensual que realizan los socios activos, se evidencia como parte fundamental la participación de los socios.

Asimismo, la participación en el mercado de depósitos a Setiembre del 2017 en miles de soles es de 203, que representa el 2,3\% a nivel nacional, de acuerdo con el estudio realizado por la Federación de Cooperativas de Ahorro y Crédito, demuestra que a pesar de que las cooperativas del país tales como: Pacífico, Abaco, Aelu y PrestaPerú, vienen a ser las mas representativas, las cooperativas Santo Domingo de Guzmán y Quillacoop acumulan una participación de mercado del 2,3\% y 2,7\%, destacando el mayor crecimiento de depósitos de la CAC Santo Domingo de Guzmán, entre los años 2013 al 2017 pasó de S/ 124 millones a S/ 203 millones lo cual indica que la Cooperativa de Ahorro y Crédito «Santo Domingo de Guzmán» del Cusco es rentable.

Para mantener una activa participación y crecimiento en el mercado financiero, es importante considerar al socio cooperativista, como elemento fundamental, que es parte de la liquidez financiera, sin su participación no es posible realizar una adecuada gestión financiera, pero también es importante mencionar que la evolución del índice de morosidad también va en aumento, de una total del 10,07\% a diciembre del 2012, se observa incremento al 14,42\% a diciembre del 2017, si viene es cierto que la cooperativa mantiene una imagen corporativa de confianza en la sociedad, aun es importante mejorar su participación en el mercado, debido a que le lleva una 
considerable ventaja a pesar de los años que viene operando en el mercado se espera mejorar y consolidar su participación.

\section{CONCLUSIONES}

Los socios cooperativistas a través de su participación vienen a conformar el pilar fundamental para la cooperativas de ahorro y crédito alcancen su objetivos y metas, tales como la participación en el mercado financiero, para lo cual requiere un mejor desarrollo de la educación financiera, esto fomenta el conocimiento de diferentes productos y servicios financieros, haciéndoles notar sus derechos y obligaciones, asimismo tener en cuenta los diferentes riesgos financieros, además de las habilidades y actitudes que ya demuestran tener, es crucial que todavía se afiance su capacidad de manejo de las alternativas crediticias, respecto a los ahorros e inversiones y la toma de decisiones para alcanzar el bienestar financiero a través de un apropiado manejo de la administración de sus finanzas personales, esto directamente influencia en la gestión financiera de la Cooperativa de Ahorro y Crédito «Santo Domingo de Guzmán» del Cusco, logrando un mejor manejo de los procesos administrativos tales como la planificación, organización, dirección y control financiero.

De acuerdo a los resultados de la regresión lineal, como parte del análisis multivariado, el modelo, indica que la dimensión conocimiento financiero -a través de una mayor comprensión del costo beneficio de los productos y servicios financieros, la gestión de los servicios financieros, riesgos financieros, así como también de los derechos y obligaciones de los socios- viene a conformar la base o columna para que la Cooperativa de Ahorro y Crédito «Santo Domingo de Guzmán» del Cusco siga incrementando su participación en el mercado y logre además la mejor liquidez financiera posible, lo cual permitirá hacer frente a las obligaciones de la cooperativa en un corto plazo.

Es importante que la Cooperativa de Ahorro y Crédito «Santo Domingo de Guzmán» del Cusco, a través de su comité de educación, considere los alcances del presente estudio, debiendo reflexionar en la forma que vienen desarrollando su gestión financiera, porque la tasa de morosidad de los socios y otros, aun no se está controlando adecuadamente, observándose un crecimiento tal vez ligeramente significativo, pero en el tiempo podría ocasionar serios problemas financieros, asimismo no se evidencia un fomento adecuado de la educación y cultura financiera en los socios, debido a esta 
situación desfavorable, muchos socios vienen retirándose y perdiendo la condición de activos, quienes finalmente son lo más beneficiados, por tanto, se pierde gran parte de capital activo.

\section{REFERENCIAS BIBLIOGRAFICAS}

Álvarez, R. (2017). Gestión financiera e indicadores de gestión de la Banca Universal Venezolana. Centro de Investigación de Ciencias Administrativas y Gerenciales, XIV(2), 338-358.

Amat, J. (2016). Del dominio del control financiero a una perspectiva cualitativa del control de gestión. Revista Facultad de Ciencias Económicas: Investigación y Reflexión, 5-11.

Amezcua García, E. L., Arroyo Grant, M. G., \& Espinosa Mejía, F. (2014). Contexto de la educación financiera en México. Ciencia Administrativa, 21-30.

Aragó, V., \& Cabedo, J. (2011). Dirección financiera de la empresa: financiación, planificación y gestión de activo corriente. Valencia: Publicacions de la Universitat Jaume I.

Banco Bilbao Vizcaya Argentaria [BBVA]. (02 de Diciembre de 2019). Educación Financiera. Obtenido de BBVA Perú: https://www.bbva.com/es/pe/solo-un-24de-peruanos-poseen-un-buen-nivel-de-educacion-financiera/

Banco de La Nación del Perú. (2017). Plan Nacional de Educación Financiera. Lima.

Bansefi. (2008). Qué es la Educación Financiera. México: Bansefi EduFinanciera.

Bernal, C. A. (2010). Metodología de la investigación (Tercera edición ed.). Colombia: Worldcolor-Prentice Hall-PEARSON.

Comisión Nacional del Mercado de Valores, Banco de España y Servicio de Formación. (2004). La psicología de las finanzas personales. Formación en Red.

Comisión Nacional para la Protección y Defensa de los Usuarios de Servicios Financieros. (2011). El ABC de la Educación Financiera. México D.F.

De Urquijo, J. (1977). Planificación financiera de la empresa. España: Ediciones Deusto.

Demirguc-Kunt, A., \& Klapper, L. (2012). Measuring Financial Inclusion: The Global Findex Database. Policy Research Working Paper, 1-58.

Federacion Peruana de Cajas Municipales de Ahorro y Credito del Perú. (2015). El microfinanciero. Revista de la Federacion Peruana de Cajas Municipales de Ahorro y Credito del Perú.

Galarza, S. P., García, J. d., Ballesteros, L., Cuenca, V. E., \& Fernández, A. (2017).

Estructura organizacional y estilos de liderazgo en Cooperativas de Ahorro y Crédito de Pichincha. Revista de Cooperativismo y Desarrollo, V(1), 19-31. 
Gitman, J. (2007). Principios de administración financiera. México: Editorial Pearson.

Hernández Sampieri, R., Fernández Collado, C., \& Baptista Lucio, P. (2014).

Metodologia de la Investigación Cientifica (Sexta ed.). México: Interamericana Editores S.A.

International Business Machines. (2008). Cómo equilibrar el riesgo y el desempeño con una organización financiera integrada. Nueva York: IBM.

León, B. (2006). Aptitud financiera y bienestar financiero de los empleados de la Universidad de Montemorelos. México: Universidad de Montemorelos.

Martínez, J. (2016). Modelo de gestión financiera basado en la optimización de las necesidades operativas de fondos: el caso de las empresas farmacéuticas en España. Madrid: Universidad Complutense de Madrid.

Organización para la Cooperación y el Desarrollo Económico. (2005). Principios y buenas prácticas para la concienciación y educación financiera. París: OCDE.

Pinar, C., \& Rivas, S. (2002). Gestión de movimiento de fondos.

Prawitz, A., Shatwell, P., Haynes, G., Hanson, K., Hanson, E., \& Garman, T. (2007). Lifestyle Risk Factors Health Status, and Financial Distress. Financial Counseling and Planning Education.

Raccanello, K., \& Herrera Guzmán, E. (2014). Educación e inclusión financiera. Revista Latinoamericana de Estudios Educativos, XLIV(2), 119-141.

Terrazas, R. (2009). Modelo de gestión financiera para una organización. Revista Perspectivas, 55-72.

Triola, M. (2004). Estadística. México: Pearson.

Trivelli, U. (2009). Objetivo de la educación financiera. Lima.

Visa Inc. (2015). Habilidades financieras prácticas, una guía útil para manejar su dinero. Canada. 